\title{
Microbiome-driven allergic lung inflammation is ameliorated by short-chain fatty acids
}

\author{
A Cait ${ }^{1}$, MR Hughes ${ }^{2}$, F Antignano ${ }^{2}, \mathrm{~J} \mathrm{Cait}^{2}$, PA Dimitriu ${ }^{1}$, KR Maas ${ }^{1}$, LA Reynolds ${ }^{3}$, L Hacker $^{2}, \mathrm{~J} \mathrm{Mohr}^{2}$, \\ BB Finlay ${ }^{1,3,4}$, C Zaph ${ }^{5,6}$, KM McNagny ${ }^{2,7}$ and WW Mohn ${ }^{1}$
}

The mammalian gastrointestinal tract harbors a microbial community with metabolic activity critical for host health, including metabolites that can modulate effector functions of immune cells. Mice treated with vancomycin have an altered microbiome and metabolite profile, exhibit exacerbated T helper type 2 cell (Th2) responses, and are more susceptible to allergic lung inflammation. Here we show that dietary supplementation with short-chain fatty acids (SCFAs) ameliorates this enhanced asthma susceptibility by modulating the activity of Tcells and dendritic cells (DCs). Dysbiotic mice treated with SCFAs have fewer interleukin-4 (IL4)-producing CD4 ${ }^{+} \mathrm{T}$ cells and decreased levels of circulating immunoglobulin E (IgE). In addition, DCs exposed to SCFAs activate T cells less robustly, are less motile in response to CCL19 in vitro, and exhibit a dampened ability to transport inhaled allergens to lung draining nodes. Our data thus demonstrate that gut dysbiosis can exacerbate allergic lung inflammation through both Tcell- and DC-dependent mechanisms that are inhibited by SCFAs.

\section{INTRODUCTION}

Short-chain fatty acids (SCFAs) are small carboxylic acids containing one to six carbon atoms. Acetic acid, propionic acid, and butyric acid are the most abundant SCFAs in the human and murine gut and are produced by bacterial fermentation of indigestible fibers and amino acids. ${ }^{1-3}$ The colons of germ-free mice have higher levels of fermentable substrates and diminished levels of SCFAs compared with those of conventionally raised mice colonized by commensal intestinal flora. ${ }^{4}$ This phenomenon highlights the essential role of the microbiome in the production of SCFAs in the gut. These SCFAs are then absorbed and circulate in the serum. Recently, there has been interest in uncovering potential roles of SCFAs in regulating the immune system.

SCFAs generated by the gut microbiome have been implicated in modulation of the immune system and in the development of allergic disease. ${ }^{5}$ For example, SCFAs were previously shown to play a role in modulating the immune response in a mouse model of allergic asthma. ${ }^{6,7}$ Similarly, we recently found that at 3 months of age, lower concentrations of fecal acetate correlated with a high propensity for the development of atopy in childhood. ${ }^{8}$

Previous work from our laboratories found that vancomycin dramatically alters the mouse gut microbiome, leading to disrupted immune homeostasis and increased susceptibility to allergic inflammation. ${ }^{9}$ Disruption of the microbiome after antibiotic treatment results in an altered metabolome with attenuated production of SCFAs. ${ }^{10,11}$ We hypothesized that a reduction of SCFA metabolites in vancomycin-treated mice contributes to their enhanced susceptibility to allergic airway inflammation.

Here, we studied the effect of vancomycin on SCFA production by the gut microbiome and the ability of exogenous SCFAs to prevent exacerbation of lung inflammation by vancomycin treatment in two models of asthma. We examined effects of SCFAs on T helper type 2 cell (Th2) skewing associated with asthma both in vivo and in vitro. Our results show that gut microbiome metabolites can have an instructive

${ }^{1}$ Department of Microbiology and Immunology, Life Sciences Institute, University of British Columbia, Vancouver, British Columbia, Canada. ${ }^{2}$ The Biomedical Research Centre, University of British Columbia, Vancouver, British Columbia, Canada. ${ }^{3}$ Michael Smith Laboratories, University of British Columbia, Vancouver, British Columbia, Canada. ${ }^{4}$ Department of Biochemistry and Molecular Biology, University of British Columbia, Vancouver, British Columbia, Canada. ${ }^{5}$ Department of Pathology and Laboratory Medicine, University of British Columbia, Vancouver, British Columbia, Canada. ${ }^{6}$ Biomedicine Discovery Institute, Department of Biochemistry and Molecular Biology, Monash University, Clayton, Victoria, Australia and ${ }^{7}$ Department of Medical Genetics, University of British Columbia, Vancouver, British Columbia, Canada. Correspondence: MR Hughes or WW Mohn (mhughes@brc.ubc.ca or wmohn@mail.ubc.ca) 
effect on critical immune cells, both T cells and dendritic cells (DCs), associated with Th2 immunity. This study provides novel mechanistic insights into the communication between the gut microbiome and immune system, the mediation of systemic health, and the maintenance of homeostasis. These insights may lead to a therapeutic approach to prevent or limit the severity of allergic asthma.

\section{RESULTS}

Vancomycin treatment depletes SCFA-producing intestinal bacteria

Our objective was to identify mechanisms downstream of vancomycin-induced gut dysbiosis responsible for promoting susceptibility to allergic asthma. To do this, we further examined the effect of vancomycin treatment on the mouse microbiome. Vancomycin-treated mice were generated from pregnant dams maintained on autoclaved drinking water containing vancomycin $\left(200 \mathrm{mgl}^{-1}\right)$ starting between day 10 and 14 of gestation. Pups were subsequently maintained on such water for life. Control mice were generated from pregnant dams maintained on autoclaved water. We analyzed the fecal microbiome by sequencing $16 \mathrm{~S}$ rRNA gene amplicons. The microbiome was significantly altered by vancomycin treatment (permutational multivariate analysis of variance results $P<0.001)$. Analyses of differential abundance of operational taxonomic units (OTUs) showed that vancomycin-treated mice had a significant reduction in OTUs belonging to several families, many of which are among the major SCFA producers in gut, namely Clostridiaceae, Lachnospiraceae, and Ruminococcaceae (Figure 1a). Notably, vancomycin treatment reduced the overall abundance, diversity, and species composition of the class Clostridia, the dominant butyrate-producing component of the intestinal microbiome (Figure 1b). ${ }^{12-15}$

To assess whether the microbiome shift led to lower SCFA production, we measured the concentrations of butyrate, propionate, and acetate in the feces and cecum of treated mice. We observed reduced levels of butyrate in the cecum and feces of vancomycin-treated mice but not of acetate or propionate compared with control mice (Figure 1c). Butyrate levels were below the limit of detection in the serum. Propionate and acetate were not significantly reduced in the serum of vancomycin-treated mice (supplementary Figure S1 online). Supplementing the drinking water of vancomycintreated mice with a mixture of butyrate, acetate, and propionate $(\mathrm{BAP})^{16}$ partially restored the concentration of butyrate detected in the cecum but did not restore butyrate in the feces (Figure 1c). These treatments did not significantly alter propionate concentrations, but the trends for propionate were similar to those for butyrate with the differences in concentration among treatments being smaller.

Because SCFAs, including butyrate, have been reported to stimulate growth of intestinal microbes, ${ }^{17}$ we assessed whether supplementing drinking water with BAP altered the fecal microbiome of mice. Hierarchical clustering based on the similarity of bacterial microbiome composition at the OTU level confirmed a major shift in composition due to vancomycin treatment with a Bray-Curtis dissimilarity index of 0.8 (Figure 1d). However, treatment with BAP did not substantially alter the microbiome of vancomycin-treated or control mice. As seen in Figure 1d, the bacterial community at the class level is dramatically altered with vancomycin treatment, but not by the addition of BAP. These results show that exogenous SCFAs (in the form of BAP) had no detectable effect on the fecal microbiome composition.

\section{SCFAs attenuate allergic lung inflammation in vancomycin- treated mice}

The ovalbumin (OVA) model of allergic asthma is a useful model to recapitulate many of the hallmarks of a Th2 celldriven lung inflammatory disease. The results from our microbiome and SCFA analyses led us to hypothesize that the reduction of bacterially derived SCFA in vancomycintreated mice makes them more susceptible to OVA-induced asthma and that exogenous SCFA (i.e., BAP water) would make them less susceptible.

As expected, mice treated with vancomycin displayed enhanced allergic inflammation with an increase of inflammatory cells and eosinophils in the lung airways and increased serum immunoglobulin E (IgE) (Figure 2a). As hypothesized, supplementation of vancomycin-treated mice with BAP in their drinking water attenuated the infiltration of inflammatory cells, including eosinophils, into the lung airways and abolished elevation of IgE. Allergic inflammation was further verified by transcript-level analysis of Th2 cytokines. We found Il4 transcript levels were significantly higher in the vancomycin treatment group relative to controls and to mice treated with vancomycin plus BAP, and trends consistent with this phenotype were observed for both Il5 and Ill3 transcripts (Supplementary Figure S2a). We examined lungs from all treatment groups for histopathology consistent with allergic inflammation and found vancomycin-treated mice had elevated pathology and increased mucus in the lungs, and this elevation was prevented by SCFA supplementation (Supplementary Figure S2b,c).

We found that butyrate supplementation, alone, was sufficient to attenuate OVA-induced airway inflammation (Figure 2b). SCFA supplementation did not significantly alter severity of airway inflammation in mice raised on control water (Figure 2c).

The OVA-allergic asthma mouse model relies on systemic antigen priming followed by induction of lung inflammation by direct airway challenge. It is possible that the influence of the intestinal microbiome and SCFAs may only be relevant in this systemic priming asthma model. Therefore, we also evaluated the effect of vancomycin and SCFAs in a model of papain-induced lung inflammation that relies entirely on repeated intranasal antigen exposure. We again evaluated asthma severity by quantifying cell infiltration into the airway and serum IgE levels. By both measures, vancomycin treatment increased susceptibly to papain-induced lung inflammation, and exogenous BAP completely prevented the enhanced inflammation attributable to vancomycin 
a

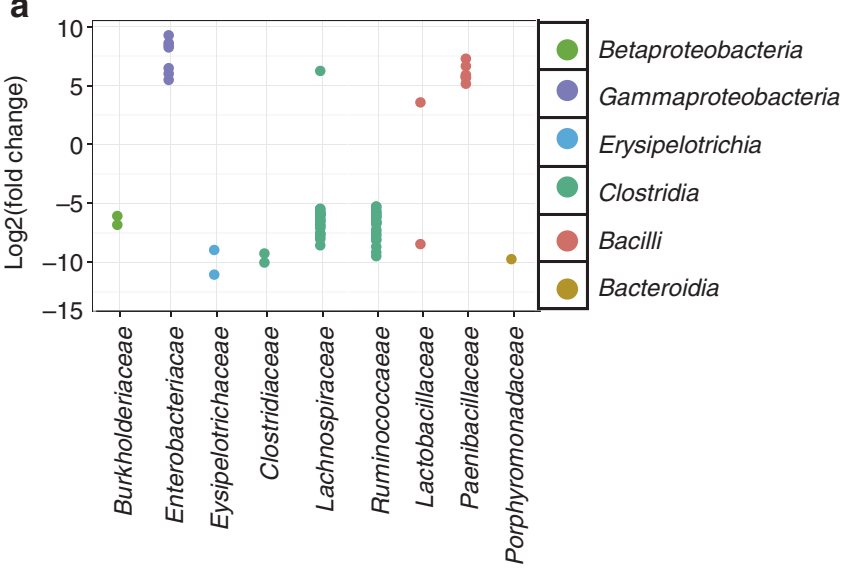

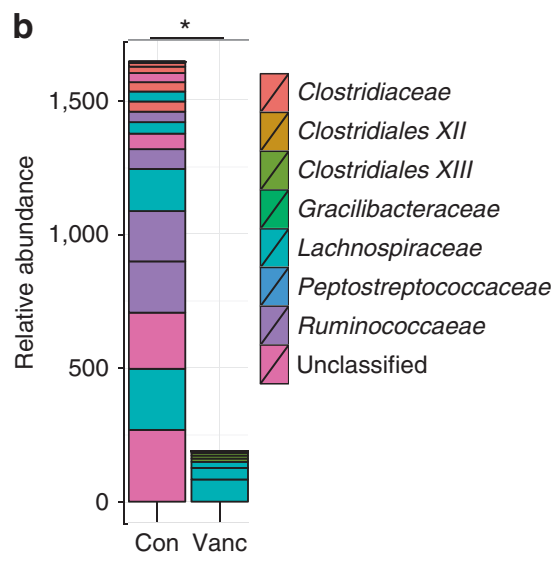

Propionate
C

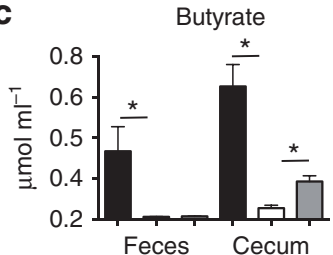

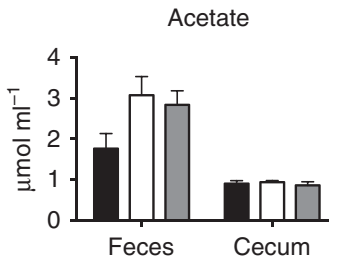

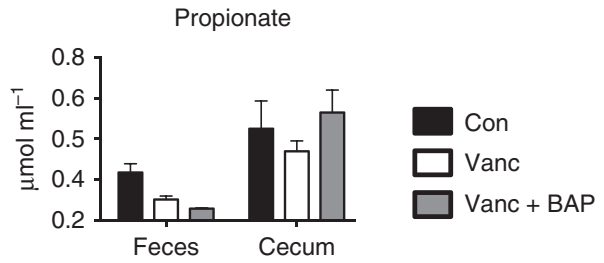

d
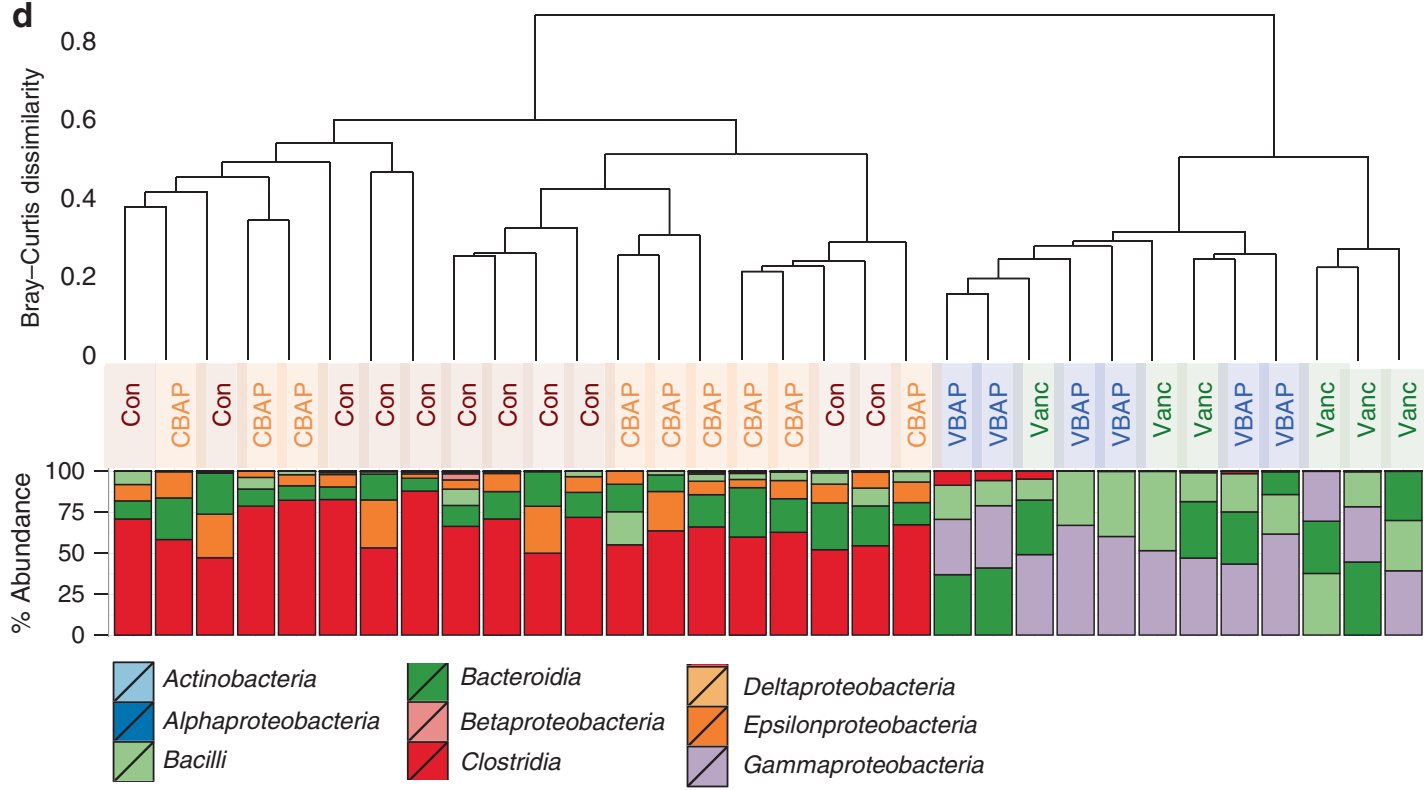

Figure 1 Vancomycin depletes short-chain fatty acid (SCFA)-producing bacteria in the gut. Mice were reared for 6-8 weeks (Con), where indicated, with vancomycin (Vanc) or Vanc + BAP in their water. (a) DESeq analysis of differentially abundant operational taxonomic units (OTUs) in control vs. vancomycin-treated mice grouped by family and color coded by class; negative fold change values indicate OTUs reduced by vancomycin, $P<0.01$ after correction for false discovery (Benjamini-Hochberg method). (b) Relative abundance of OTUs belonging to the class, Clostridia, color coded by family. (c) Concentration of the three most abundant SCFAs measured in cecum and feces of mice, measured by gas chromatography, and normalized to volume of sample. (d) Community composition at the class level of each sample is shown with hierarchical clustering based on Bray-Curtis dissimilarity of OTU profiles. Drinking water treatments of mice: Con, control; Vanc, vancomycin; Vanc + BAP or VBAP, vancomycin plus butyrate, acetate, and propionate. Samples from replicate experiments were combined for analysis $(n=6)$; error bars show s.e.m. ${ }^{\star} P<0.05$.

(Figure 2d). Allergic inflammation was further verified by transcript-level analysis of Th2 cytokines. We found Il13 transcript levels were significantly higher in the vancomycin treatment group, and trends consistent with this were seen for both Il4 and Il5 (Supplementary Figure S2d). We examined lungs from all treatment groups for histopathology consistent with allergic inflammation and found vancomycin-treated mice had elevated pathology and increased mucus in the lungs relative to controls. Mice on vancomycin supplemented with SCFAs did not have elevated pathology scores or mucus production (Supplementary Figure S2e,f). To further understand how BAP and vancomycin treatment influences 
a
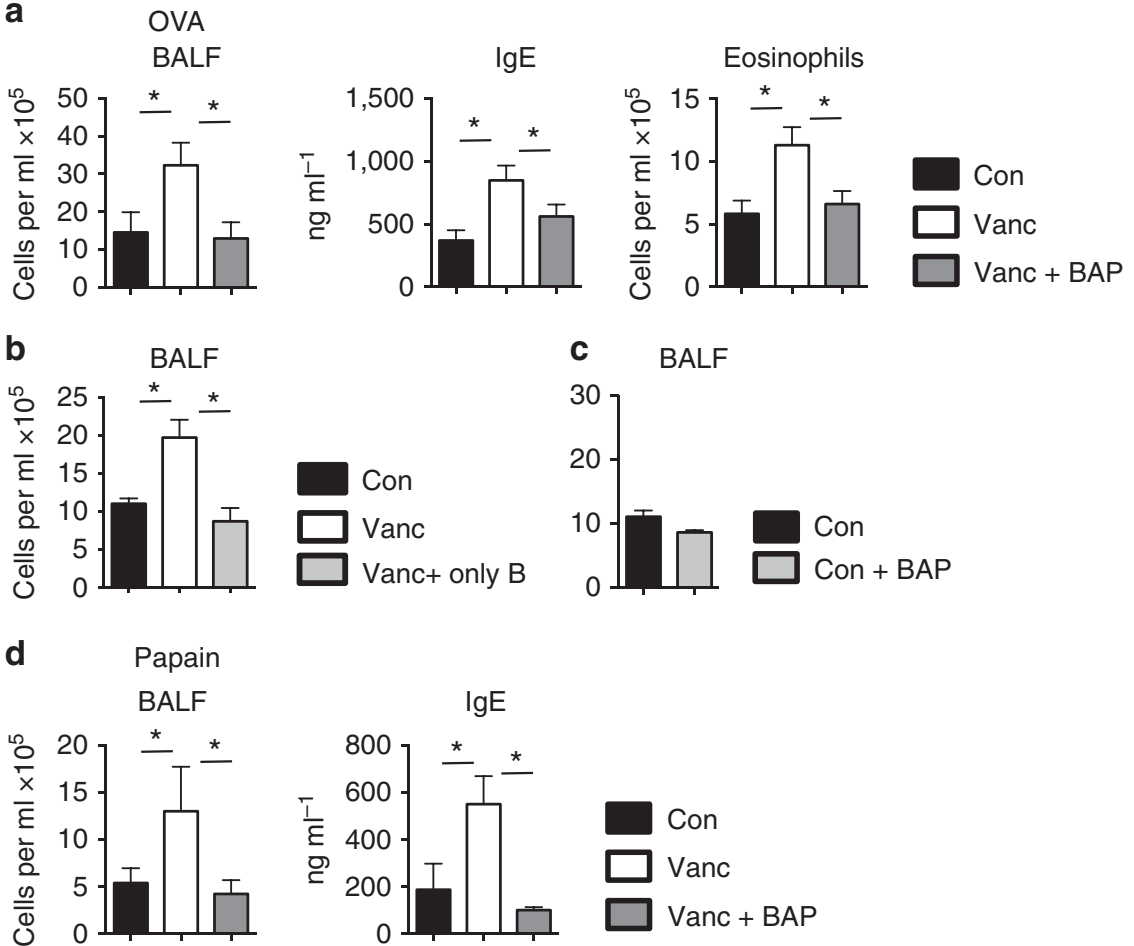

Figure 2 Exogenous short-chain fatty acids (SCFAs) ameliorate vancomycin-induced asthma severity. (a-c) Assessment of asthma severity in ovalbumin (OVA)-treated mice. (a) Total cell counts in the bronchiolar lavage fluid (BALF), serum level immunoglobulin $E$ (IgE), and total number of eosinophils in BALF. (b) Total cell counts in the bronchiolar lavage of OVA-induced mice; Vanc $+B$, treated with vancomycin plus only butyrate. (c) Total cell counts in the bronchiolar lavage of OVA-treated mice; control water vs. control water supplemented with butyrate, acetate, and propionate (BAP). (d) Assessment of asthma severity in papain-treated mice: total cell counts in the BALF, and serum level IgE. The data in a come from 3 independent experiments with 4 to 6 mice per group, data in $\mathbf{b}, \mathbf{c}$ come from 2 independent experiments with 4 to 6 mice per group. In a-c, data show a representative experiment. Data from $\mathbf{d}$ are from 4 independent experiments with 5 mice per group, samples from replicate groups were combined for data analysis. Error bars in all panels show s.e.m. ${ }^{*} P<0.05$.

IgE levels, we looked for evidence of IL4-driven IgE isotype switching in lymphoid tissues following papain induction. Expression of the sterile germline transcript ( $\varepsilon$ GLT) in B cells is indicative of ongoing isotype switching to $\operatorname{IgE} .^{18}$ We found $\varepsilon$ GLT to be significantly higher in mesenteric lymph nodes (LNs), Peyer's patches (PPs), ${ }^{18}$ and mediastinal LNs of vancomycin-treated mice, while this elevated transcription was prevented by BAP (Supplementary Figure S3).

\section{SCFAs attenuate vancomycin-induced IgE production}

To help determine the mechanisms by which SCFAs attenuate lung inflammation, we evaluated antibiotic-treated but otherwise naive mice (not exposed to OVA or papain) for indicators of a helper $\mathrm{T}$ cell polarization bias. We first examined the naive lung for elevated levels of Th2 cytokines, immune cell infiltrate, and histopathology and found no indications of inflammation due to vancomycin treatment (Supplementary Figure S4). We next looked systemically for a marker of Th2 polarization bias, elevated serum IgE. Vancomycin-treated mice had significantly elevated levels of circulating IgE, but not IgA or $\operatorname{IgG}_{2 \mathrm{a}}$ compared with control mice, and BAP supplementation attenuated this elevated IgE production (Figure 3a).

We additionally looked for evidence of isotype switching to IgE in the naive mice by measuring eGLT expression in lymphoid tissues. In vancomycin-treated mice, eGLT expression was not substantially elevated in the spleen compared with control mice but was dramatically elevated in $\mathrm{PPs}^{18}$ (Figure 3b). These findings are consistent with the IL4driven class switching observed in the PPs of germ-free mice. ${ }^{19}$ In fact, the elevated serum IgE in germ-free mice is largely dependent on the development of $\mathrm{PPs},{ }^{19}$ suggesting that PPs are a major source of circulating IgE in naive mice. Importantly, supplementation with BAP prevented the elevation of EGLT expression in the PPs of vancomycintreated mice. Finally, we found that the maximal dampening of IgE levels in vancomycin-treated mice occurred only if SCFA supplementation was introduced at or before weaning (3 weeks) (Figure 3c).

\section{SCFAs attenuate vancomycin-induced IL4 production}

Interleukin-4 (IL4) plays a pivotal role in shaping the environment of the immune response in allergic disease by promoting isotype switching from IgM to IgG1 and IgE. Th2polarized T cells are an important source of IL4 (in addition to other cell types including basophils). ${ }^{20}$ Using an IL4-GFP reporter mouse strain (4get mice), ${ }^{21}$ we determined whether $\mathrm{CD} 4{ }^{+} \mathrm{T}$ cells from vancomycin-treated mice were predisposed to producing IL4. We could only detect low levels of IL4 expression in $\mathrm{T}$ cells taken directly from the spleen of naive 4get mice reared on vancomycin water or control water (not shown). 
a

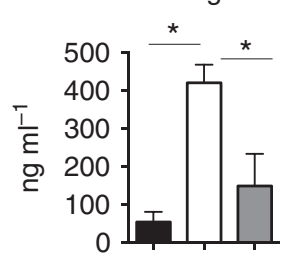

b Spleen $\varepsilon G L T$

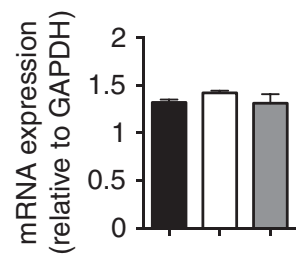

$\lg \mathrm{A}$

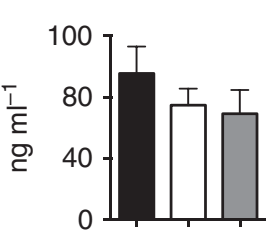

$\mathrm{PP} \varepsilon \mathrm{GLT}$

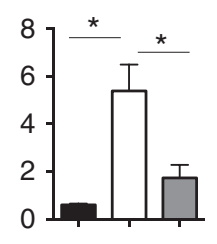

$\lg \mathrm{g} 2 \mathrm{a}$
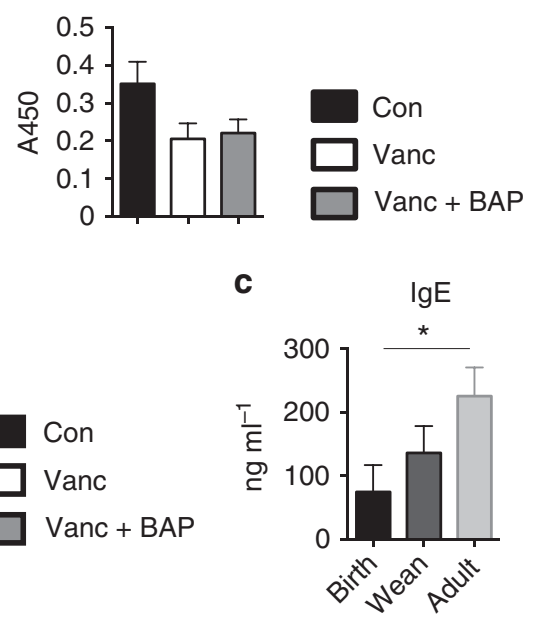

Figure 3 Short-chain fatty acid (SCFA) supplementation (butyrate, acetate, and propionate (BAP)) attenuates vancomycin-induced immunoglobulin E $(\mathrm{IgE})$ production. Naive mice were reared for 8 weeks. (a) Concentrations of circulating antibodies in blood serum of naive mice. Con, control; Vanc, vancomycin. (b) Transcript levels of $\varepsilon G L T$ measured in spleen normalized to the housekeeping gene, glyceraldehyde 3-phosphate dehydrogenase (GAPDH). (c) Circulating IgE in blood serum of vancomycin-treated mice with exogenous BAP commencing at birth, at weaning (3 weeks), or at adulthood (7 weeks). Data from a are from 3 independent experiments with 4-6 mice per group, samples from replicate groups were combined for data analysis. Data from $\mathbf{b}$ are from 3 independent experiments with 5 mice per group, one representative experiment is shown. Data from $\mathbf{c}$ are from 2 independent experiments with 5-6 mice per group, samples from replicate groups were combined for data analysis. Error bars in all panels show s.e.m. ${ }^{\star} P<0.05$.

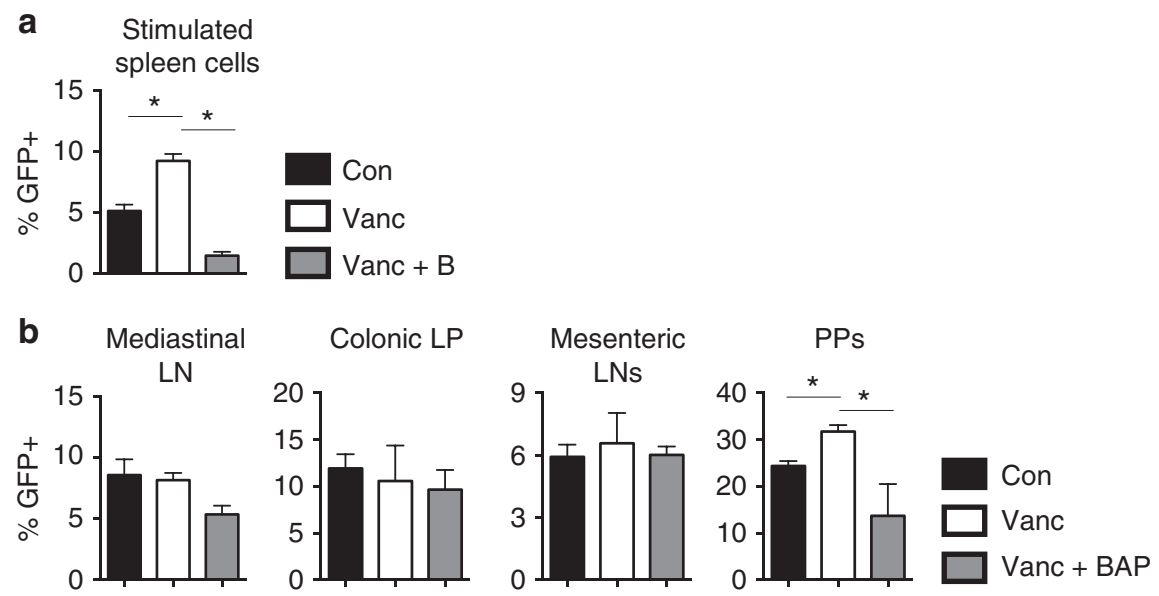

Figure 4 Short-chain fatty acids (SCFA) attenuate vancomycin-induced interleukin-4 (IL4) production. Percentage of CD4 + T cells from naive 4get mice producing IL4 gene expression by green fluorescent protein (GFP) expression via flow cytometry. (a) Cells from spleens of mice incubated in vitro under T helper type 2 cell (Th2)-stimulating conditions; mice were treated as in Figure $\mathbf{2 b}$ (i.e., B, only butyrate). (b) In vivo expression in unstimulated cells from lymph nodes as indicated. Data from a are from 3 independent experiments with 3 mice per group, one representative experiment is shown. Data from $b$ are from 2 independent experiments with 5-6 mice per group, samples from replicate groups were combined for data analysis. Error bars in all panels show s.e.m. ${ }^{*} P<0.05$. LN, lymph node; LP, lamina propria; PP, Peyer's patches.

However, when stimulated ex vivo under Th2 cell-polarizing conditions, splenocytes harvested from vancomycin-treated 4get mice yielded a higher proportion of GFP-positive $\mathrm{CD} 4^{+} \mathrm{T}$ cells than splenocytes from control mice (Figure 4a). Furthermore, butyrate supplementation in vitro reduced the proportion of IL4-producing $\mathrm{CD} 4{ }^{+} \mathrm{T}$ cells from vancomycintreated mice to below that of control mice. These results indicate that butyrate inhibits IL4 expression in T cells.

We next assessed the effects of vancomycin and SCFAs on IL4 expression in T cells in vivo using the 4get mice. Consistent with the IgE class switching activity we observed in the PPs of vancomycin-treated mice, we also observed in these mice an increase in the number of IL4-producing $\mathrm{CD} 4{ }^{+} \mathrm{T}$ cells in the PPs but not in the colonic lamina propria, mesenteric LNs, or mediastinal LNs (Figure 4b). Furthermore, supplementation with BAP caused a dramatic decline in the number of IL4producing $\mathrm{CD} 4{ }^{+} \mathrm{T}$ cells in the PPs but not in the other tissues we examined.

Because previous reports have focused on the role of SCFAs in regulatory $\mathrm{T}$ cell (Treg) polarization we also examined levels 
of Tregs in the mesenteric LNs, colonic lamina propria, PPs, spleen, and lung in naive mice. In mesenteric LNs and colonic lamina propria we found lower levels of Tregs in the vancomycin-treated mice relative to controls (Supplementary Figure S5). BAP supplementation prevented the decrease in Tregs in these tissues. A similar trend was observed in the PPs, but the numbers of Tregs were not statistically different. We did not observe altered levels of Tregs in the spleen and lung relative to control mice by treatment with vancomycin or vancomycin plus BAP.

\section{SCFA exposure alters the gene expression profile of DCs} We next investigated the immune processes that occur after the introduction of allergen. DCs play an essential role in the initial recognition of antigen and provide a critical link between the innate and adaptive branches of the immune response. These cells are mainly responsible for the uptake and transfer of the allergen from the airway to the local (mediastinal) LNs for presentation to and activation of $\mathrm{T}$ cells in initiation of an adaptive response. ${ }^{22,23}$ SCFAs have previously been shown to have anti-inflammatory effects on DCs. In the presence of butyrate, both human and mouse DCs express lower levels of costimulatory molecules and lower levels of inflammatory gene transcripts. ${ }^{24}$ Because DCs have previously been shown to be both necessary and sufficient for the induction of a robust Th2 cell-mediated immune response, ${ }^{25-27}$ we investigated whether the presence of SCFAs altered the ability of DCs to prime Th2-mediated immunity. To look broadly at these mechanisms, we used a transcriptomic approach.

By injecting control mice with Flt3L (FMS-like tyrosine kinase 3 ligand)-expressing B16 melanoma tumors we were able to harvest large numbers of splenic DCs for ex vivo culture. $^{28-31}$ Flt3L-induced splenic DCs were isolated by immunomagnetic bead selection, incubated ex vivo in the presence or absence of butyrate and stimulated with lipopolysaccharide (LPS). Of the 15,183 genes represented in the transcriptome, transcript levels of only 74 genes were significantly altered by butyrate exposure in LPS-activated DCs (butyrate treatment primarily dampened upregulation of these genes) (Figure 5a,b). Using Ingenuity Pathway Analysis $^{30}$ we were able to integrate expression profiles for both down- and up-regulated genes into known biological pathways. This analysis revealed a strong immunomodulatory effect of butyrate on two pathways known to affect susceptibility to allergic disease. First, we found a significantly altered expression profile suggesting that butyrate decreases the ability of DCs to activate lymphocytes (Figure 5c). Although our transcriptome data identified a mix of both down and up-regulated genes after butyrate treatment, the overall pattern indicated decreased function of the pathway (i.e., some genes will decrease T-cell activation when upregulated, and some will decrease activation when downregulated). Second, we found a profile suggesting that butyrate dampens DC trafficking mechanisms (Figure 5d).

\section{SCFAs attenuate DC activation}

To verify the pathways implicated by transcriptome analysis, we first incubated Flt3L-elicited splenic DCs with SCFA ex vivo. Incubation with butyrate did not affect DC viability (Figure 6a). After stimulation with LPS, however, Flt3L-derived DCs incubated with butyrate displayed attenuated expression of costimulatory molecules CD80 and CD86 (Figure 6b). Furthermore, DCs isolated from vancomycin-treated mice displayed increased expression of CD80 and CD86 after stimulation with LPS (Figure $\mathbf{6 c}, \mathbf{d}$ ) that could be attenuated by oral BAP supplementation. Our results are consistent with previous work suggesting that human monocyte-derived and mouse bone marrow-derived butyrate-treated DCs are less mature and express lower levels of costimulatory molecules. ${ }^{24,32-34}$ DCs from control mice were isolated and cultured $+/-$ butyrate in culture media for $3 \mathrm{~h}$. These were then washed, pulsed with OVA, and incubated with CFSE (carboxyfluorescein succinimidyl ester)-labeled OTII T cells. We found that untreated DCs activated T cells, thus inducing more proliferation, with greater efficiency than those that were preincubated with butyrate (Figure 6e). Thus, butyrate has a cell-intrinsic effect on DC activation and ability to stimulate the adaptive immune response following exposure.

\section{SCFAs attenuate DC chemotaxis}

As the CCL19/21 and CCR7 chemokine axes mediate DC migration from inflamed tissue to collecting $\mathrm{LNs},{ }^{35}$ we tested whether this migration is altered by exposure of Flt3L-elicited splenic DCs to butyrate in vitro. In a transwell chemotaxis assay, DCs harvested from control mice and incubated with butyrate for $3 \mathrm{~h}$ migrated less efficiently in response to CCL19 than corresponding DCs incubated without butyrate (Figure 7a). Furthermore, cells isolated from vancomycintreated mice migrated with greater efficiency than DCs isolated from control mice or mice treated with vancomycin plus BAP (Figure 7b). Our results show that butyrate attenuates CCL19induced DC migration.

We next tested whether the trafficking behavior of lung tissue DCs is altered in dysbiotic mice in vivo using the papain inflammatory model. In order to track DCs in vivo we included DQ-OVA in our papain challenge. DQ-OVA fluorescently labels antigen-presenting cells after phagocytosis and proteolytic processing. Upon necropsy, we enumerated DCs that had acquired antigen (DQ-OVA) in the lung and subsequently migrated to the local draining LN (mediastinal). Vancomycintreated mice had significantly higher numbers of DCs in the mediastinal LN compared with controls. Furthermore, exogenous BAP reduced trafficking of DCs in vancomycin-treated mice to levels comparable to control mice (Figure 7c). We conclude that both the sensitivity to activation and the migratory behavior of DCs is altered by SCFA exposure.

\section{DISCUSSION}

It has been widely recognized that there is a relationship between antibiotic use and the development of asthma. ${ }^{36-38}$ Our study provides novel insight into how SCFAs, as a product 
a

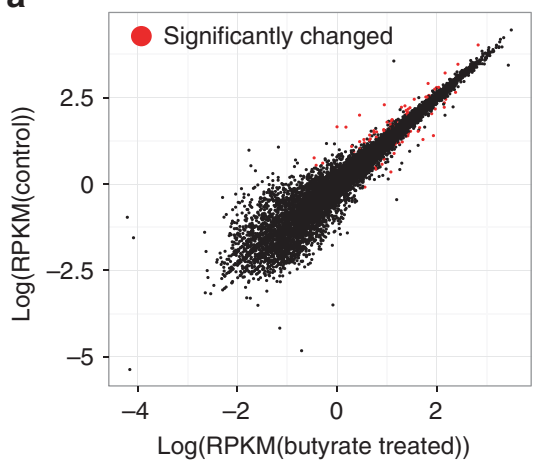

c

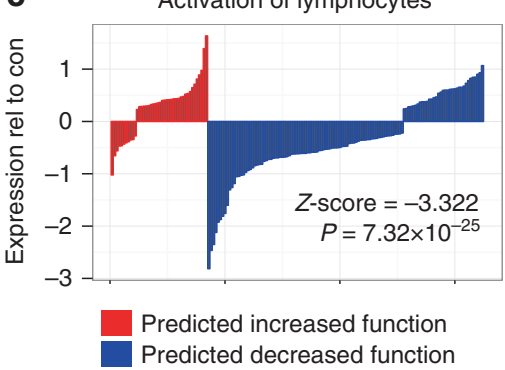

d

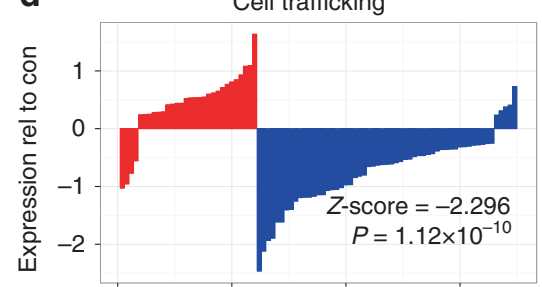

Predicted increased function

Predicted decreased function b

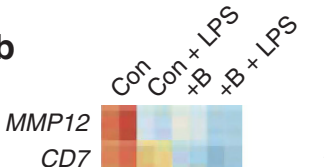

PGLYRP

NPR1

TNFRSF9

DHRS4

TREM2

SELL

PLAUR

$L P L$

CDKN2B

SOCS1

VIM

CCL22

ISG15

SERPINB9

IL6

PSME2

$C D 40$

$C D 86$

CDKN1A

NFKB1

ICAM1

NFKBIB

IL1B

CCR7

CD80

GNAI3

GADD $45 B$

$D A X X$

STAT5A

LMAN2

TRAF1

IRF8

NFKBIA

TNFRSF1B

IL12B

CLIC4

NFKBIZ

EIF4EBP2

HDAC5

IL1R1

IL18R1

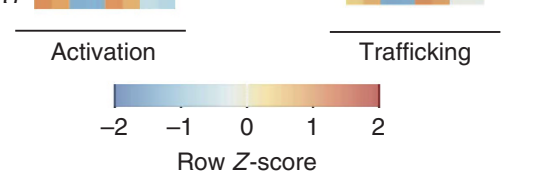

Figure 5 Transcriptomes reveal anti-inflammatory effect of butyrate on dendritic cells (DCs). (a) Log ratio of RPKM (reads per kilobase of transcript per million mapped reads) values of lipopolysaccharide (LPS)-stimulated DCs, not treated (control (Con)), or treated with butyrate (B). Transcripts in red were found to differ significantly $(P<0.05)$. (b) Heatmap showing relative expression of genes involved in cell migration and chemotaxis. Log ratio of RNA expression of genes involved in (c) lymphocyte activation and (d) cell trafficking. Color coding indicates genes whose expression is predicted to increase or decrease with the corresponding function. DCs from two control mice were subjected to four treatments. Data are pooled in $\mathbf{a}$ and $\mathbf{c}$ and individual duplicates are shown in $\mathbf{b}$.

of a specific subset of the microbiome, may serve as a mechanistic link for this association, and highlights the importance of a healthy microbiome in dampening asthma susceptibility or severity. We provide evidence that the exacerbation of OVA-induced allergic lung inflammation by vancomycin treatment is due to depletion of gut microbiome populations responsible for the production of SCFAs, particularly butyrate. Consistent with this observation, supplementation of vancomycin-treated mice with exogenous SCFAs partially restores butyrate levels in the cecum, and completely attenuates the exacerbation of lung inflammation by vancomycin in two mechanistically distinct asthma models. 


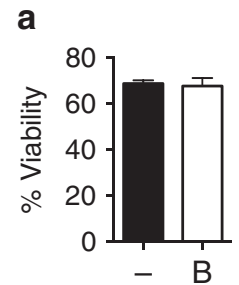

b

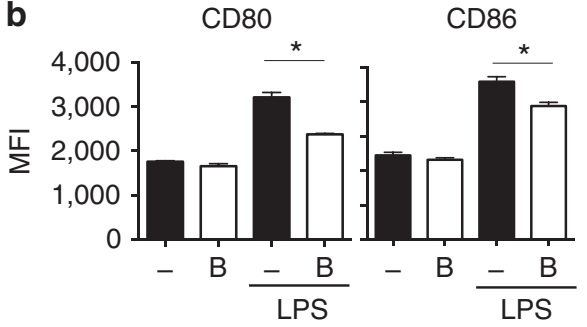

c
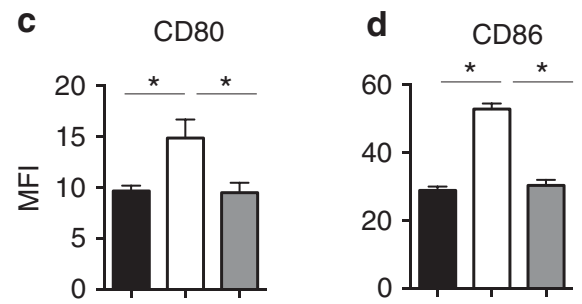

e \% Proliferated relative to

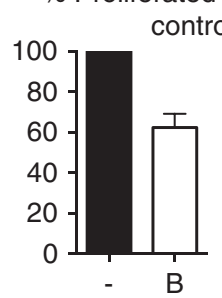

Figure 6 Short-chain fatty acids (SCFAs) attenuate dendritic cell (DC) activation. Flt-3L-derived DCs were harvested from naive mice. (a) Viability of DCs after incubating for $48 \mathrm{~h}$, without $(-$ ) or with butyrate (B). (b) Expression levels by DCs of costimulatory molecules CD80 and CD86 after stimulation with lipopolysaccharide (LPS) in vitro, as determined via mean fluorescent intensity (MFI) ${ }^{30}$ by flow cytometry. Results are expressed as fold change from the paired control cells, incubated with no LPS or butyrate. (c) Expression of costimulatory molecules CD80 and (d) CD86 by DCs isolated from mice with drinking water treatments as in Figure 1c, and DCs subsequently stimulated ex vivo with LPS for $18 \mathrm{~h}$. (e) Activation of $\mathrm{T}$ cells by DCs. DCs from control mice were isolated and cultured without ( - ) or with B in culture media for $3 \mathrm{~h}$, washed, pulsed with ovalbumin (OVA), and incubated with CFSE-labeled OTII T cells.

Proliferation of $\mathrm{CD} 4{ }^{+} \mathrm{T}$ cells was assessed by flow cytometry and percent proliferation was assessed relative to its paired no-butyrate control. Data from $\mathbf{a}$ and $\mathbf{b}$ are from 3 independent experiments with 3 mice per group, samples from replicate groups were combined for analysis. Data from $\mathbf{c}$ are from 2 independent experiments, one representative experiment is shown. Data from $\mathbf{d}$ are from 2 independent experiments, samples from replicate groups were combined for analysis. Error bars in all panels show s.e.m. ${ }^{*} P<0.05$. Con, control; Vanc, vancomycin; Vanc + BAP vancomycin plus butyrate, acetate, and propionate.

In contrast to other reports, ${ }^{6,7}$ we did not find evidence that SCFAs alter asthma severity in the presence of a healthy microbiome. We postulate that the effect of SCFAs is highly dependent on the environment (animal unit) and the variation that exists in a "normal" microbiome and SCFA levels. Similar to our findings, Arpaia et al. ${ }^{39}$ found that the oral administration of SCFAs did not have an effect on the generation of Tregs in the presence of a healthy microbiome. Although we cannot rule out the possibility that additional aspects of the gut microbiome other than SCFA production can attenuate asthma, we show here that SCFAs are sufficient to completely prevent the exacerbation of lung inflammation because of vancomycin treatment. Notably, SCFAs did so without altering the dysbiotic microbiome composition resulting from vancomycin treatment. Our results also provide evidence that the effect of vancomycin in the two models of asthma is via its effect on the microbiome and not via some direct effect of the antibiotic on the host.

The current paradigm for the function of the gut microbiome in influencing Th2-mediated disease focuses predominantly on the induction of Tregs. Our study provides novel evidence for two additional and distinct processes by which SCFAs link the gut microbiome to the immune response in asthma. We show that vancomycin treatment leads to an increase in IL4producing $\mathrm{CD}^{+} \mathrm{T}$ cells in mice naive to specific antigen that consequently promotes IgE class switching activity in the PPs. Supplementation of vancomycin-treated mice with exogenous SCFAs dampens the induction of IL4-producing $\mathrm{CD} 4{ }^{+} \mathrm{T}$ cells and class switching activity in the PPs. In addition our in vitro data show that exposure of splenic cells to butyrate under Th2 cell-polarizing conditions restrains the development of IL4-producting CD4 ${ }^{+} \mathrm{T}$ cells. Our findings are consistent with observations by others that serum IgE levels are elevated in germ-free ${ }^{19}$ and antibiotic-treated mice. ${ }^{40}$ We propose that elevated IgE in both of these models is because of reduced SCFA production in the gut. ${ }^{4}$ Interestingly, we found that the ability of SCFA to reduce IgE levels in vancomycin-treated mice was greatest if SCFAs were administered before weaning. This finding supports the hypothesis that there is a perinatal window during which the microbiome plays a key role in the development of allergic susceptibility, and that effective interventions must be started before this critical window closes. $^{8-9,41-43}$

It was previously shown that SCFA have a $\mathrm{T}$ cell-autonomous effect on the extrathymic generation of Tregs via histone modification. ${ }^{18,39}$ Our findings suggest a novel effect of SCFAs on T-cell fate: that SCFAs, butyrate in particular, may also restrain T-cell polarization towards a Th2 cell fate. Both the previously described Treg phenotype and our proposed mechanism would contribute toward reducing asthma susceptibility. We have shown that in a naive state, SCFAs play an instructive role in setting up a healthy immune environment. The lack of SCFAs primes the host for the later development of allergy by facilitating a Th2-skewed immune environment.

We found that the effects of SCFAs on the development of Th2-skewed T cells are systemic (spleen) but most dramatic in the PPs. Although this may seem counterintuitive (SCFA production begins in the cecum), it is consistent with data from several independent studies. Others have found that in a germfree mouse, the IgE levels are most dramatically affected in the PPs. ${ }^{19,44}$ The strength of this T-cell phenotype in the PPs may be explained by the fact that specific IgE responses originate in this tissue. ${ }^{45}$

In addition, we identified a second DC-dependent immunomodulatory effect of SCFAs that is consistent with an attenuated response to airway allergens in vivo. Consistent with previous observations, we found that SCFAs attenuate DC activation in vitro at the transcription level and at the protein 

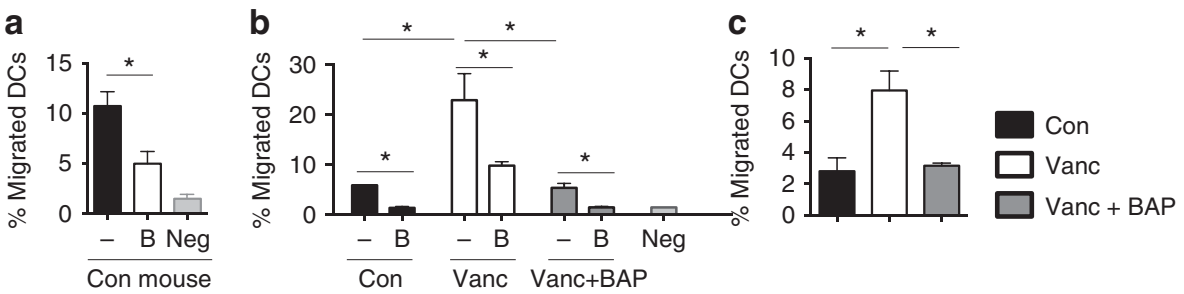

Figure 7 Short-chain fatty acids (SCFAs) attenuate dendritic cell (DC) migration. (a) Flt-3 derived DCs were harvested from naive mice, pooled, and split into treatment groups: incubated with butyrate (B) or without butyrate $(-)$ in the culture media; or with no CXCL19 (Neg). The percentage of total $\mathrm{CD}_{11 \mathrm{C}^{+}}$cells plated that migrated in a CXCL19 chemokine gradient were determined using a transwell assay and flow cytometry. (b) Flt-3 derived DCs were harvested from mice treated as in Figure 1c. Cells from each mouse were split into two treatment groups: incubated with (B) or without $(-)$ butyrate in the culture media. The percentage of DCs that migrated to a CXCL19 chemokine gradient was determined using a transwell assay and flow cytometry. Data are expressed as a percentage of total DCs plated. (c) In vivo experiment enumerating DC migration after DQ-ovalbumin (OVA)/papain challenge. DCs were from mice treated as in Figure 1c. Percentage of DQ-labeled DCs in the lung draining (mediastinal) lymph node 3 days after intranasal DQOVA/papain challenge. Data are expressed as a percentage of total CD11c + DQ-labeled cells counted in both the lung and lymph node. Data from a-c are from 2 independent experiments with 4-6 mice per group, samples from replicate groups were combined for analysis. Error bars in all panels show s.e.m. ${ }^{\star} P<0.05$. Con, control; Vanc, vancomycin; Vanc + BAP vancomycin plus butyrate, acetate, and propionate.

level. Although these experiments were done on splenic DCs, we believe that this is likely true for lung DCs as well based on functional data for lung migration and asthma severity. For the first time, we demonstrate that a lack of SCFA exposure in vivo has a persistent effect on DC activation. Furthermore, we demonstrate that SCFA exposure in vivo dramatically attenuates DC migration and that butyrate attenuates DC migration and activation in vitro. Attenuation of DC migration by SCFAs could have far reaching consequences for many diseases, including allergic disease, autoimmune disease, and infection.

In summary, we find that SCFAs modulate the systemic immune response by dampening $\mathrm{Th} 2$ responses through direct effects on both T cells and DCs. These findings highlight the importance of assessing the appropriate use of antibiotics, especially in young children, and identify potential approaches for the development of probiotics or metabolite therapy to prevent childhood asthma and other Th2-driven disease.

\section{METHODS}

Mice. C57BL/6J and C.129-IL4tm1Lky/J (4get) mice (The Jackson Laboratory, Bar Harbor, ME) were bred and maintained in a specific pathogen-free facility at the Biomedical Research Centre (Vancouver, BC, Canada). Mice were housed in autoclaved cages and received irradiated chow ad libitum (equal parts mixture of PicoLab Mouse Diet 20 and Picolab Rodent Diet 20 (St. Louis, MO)) and autoclaved tap water. All experiments were carried out in accordance with the Canadian Council on Animal Care guidelines and were approved by the University of British Columbia Committee on Animal Care (protocol no. A15-0113). At experimental end points mice were humanely killed by avertin (2,2,2-tribromoethanol) overdose followed by cardiac puncture or removal of lungs.

Antibiotic and SCFA treatment and quantification. As indicated, breeding pairs and nursing dams were administered vancomycin (Sigma-Aldrich, St Louis, MO) at $200 \mathrm{mgl}^{-1}$ in drinking water. Pups born from respective breeding pairs were reared on antibiotic-treated water for the duration of the experiment. As indicated, a cocktail of SCFA, $40 \mathrm{~mm}$ butyrate, $67.5 \mathrm{~mm}$ acetate plus $25.9 \mathrm{~mm}$ propionate (Sigma-Aldrich), was included in drinking water. Alternatively, $40 \mathrm{~mm}$ butyrate was included. SCFA solutions were prepared and changed weekly. To quantify SCFA levels, mouse fecal and cecal samples were combined by vortexing with $25 \%$ phosphoric acid followed by centrifugation until a clear supernatant was obtained. Supernatants were submitted for GC analysis to the Department of Agricultural, Food and Nutritional Science of the University of Alberta (Edmonton, $\mathrm{AB}$, Canada). Samples were analyzed as previously described. ${ }^{8}$

Microbiome analysis. Fecal pellets were collected from individual mice and stored at $-70^{\circ} \mathrm{C}$. Pellets were homogenized using a beadbeating method (FastPrep bead matrix E, MP Biomedicals, Santa Ana, CA), and total DNA was extracted (Ultra Clean Fecal DNA kit, Mo Bio Laboratories, Vancouver, BC). The $16 \mathrm{~S}$ rRNA gene fragments were amplified using bar-coded primers, ${ }^{8}$ with the following primer regions $\left(5^{\prime}-3^{\prime}\right)$ : 27F: AGAGTTTGATCMTGGCTCAG, 519R: GWATTACCGCGGCKGCTG. Amplicons were pooled in equal molar concentrations and pyrosequenced using a 454 Titanium platform (Roche, Basel, Switzerland). Sequence data were trimmed, quality filtered, and clustered at $97 \%$ identity into OTUs using a modified MOTHUR standard operating procedure. ${ }^{46}$ OTUs were taxonomically annotated using the SILVA database. ${ }^{47}$ Global community structure comparisons were made using DESeq2 with an adjusted (BenjaminiHochberg method) $P$ value of $<0.01$ and Phyloseq. ${ }^{48}$

Antibodies and flow cytometry. Staining and antibody dilutions were prepared in phosphate-buffered saline containing $2 \%$ fetal calf serum, $2 \mathrm{~mm}$ EDTA, and $0.05 \%$ sodium azide. Samples were first blocked in buffer containing $5 \mathrm{mg} \mathrm{ml}^{-1}$ anti-CD16/32 (clone 2.4G2). Antibodies used were as follows: PE-conjugated Siglec-F (E50-2440) from BD Biosciences (San Jose, CA), PE-Cy7-conjugated CD3e from eBioscience (San Diego, CA), fluorescein isothiocyanate-conjugated anti-neutrophil (7/4) from Abcam (Cambridge, MA), Alexa Fluor 647-conjugated CD4 (RM4-5) from eBioscience (Santa Clara, CA), Pacific Blue-conjugated CD45 (I3/2) made in-house, and Alexa Fluor 647-conjugated CD11c (N418) made in-house. Samples were run on a BD LSRII, and data analysis was performed with FlowJo software (TreeStar, CA).

OVA-induced allergic lung inflammation. On days 0 and 7 , mice were injected intraperitoneally with $50 \mathrm{mg}$ of chicken OVA (grade III; Sigma-Aldrich) and $650 \mathrm{mg}$ of aluminum hydroxide (Sigma). On days $21,22,23,25$, and 27 , mice were intranasally challenged with $50 \mathrm{mg}$ of OVA. On day 28 , mice were killed by avertin overdose. Bronchoalveolar lavage fluid was collected by 3 washes with $1 \mathrm{ml}$ of sterile saline. Blood was collected by cardiac puncture. Cells were enumerated and differentiated by flow cytometry using antibodies to CD3e, CD11c, CD45, B220, Siglec-F, and 7/4.

Papain-induced allergic lung inflammation. On days $0,1,14$, and 20, lightly anesthetized mice were administered intranasally $10 \mu \mathrm{g}$ of papain (Sigma) prepared in a volume of $40 \mu \mathrm{l}$ phosphate-buffered 
saline. Experimental mice were killed $16 \mathrm{~h}$ after the final papain treatment (on day 21). Bronchoalveolar lavage fluid was collected by 3 washes with $1 \mathrm{ml}$ of sterile saline. Blood was collected by cardiac puncture. Cells were enumerated and differentiated by flow cytometry using antibodies to CD3e, CD11c, CD45, B220, Siglec-F, and 7/4.

RNA isolation and quantitative reverse transcriptase-PCR. Using a Qiagen TissueLyser (Valencia, CA), tissues were homogenized in Trizol (Life Technologies, Carlsbad, CA). Total RNA was extracted and reversed transcribed with a high-capacity complementary DNA kit (Life Technologies). Quantitative PCR was performed with SYBR green technology (KAPA Biosystems, St. Louis, MO) on an ABI 7900 real-time PCR instrument (Life Technologies). Primer sequences were as follows $\left(5^{\prime}-3^{\prime}\right)$ : $\varepsilon$-GLT: forward GCCTGCACAGGGGGCAGAAG and reverse ATGACCCTGGGCTGCCTGGT, GAPDH: forward CATCAAGAAGGTGGTGAAGC and reverse CCTGTTGCTGTAG CCGTATT, $\beta$-actin forward ACTAATGGCAACGAGCGGTTC and reverse GGATGCCACAGGATTCCATACC.

Histology. The left lung lobe was fixed in $10 \%$ buffered formalin, embedded in paraffin, and stained with hematoxylin and eosin or periodic acid-Schiff. Hematoxylin and eosin-stained sections were blindly scored on a scale of 0 to 12 as previously described. ${ }^{49}$ Mucus was quantified on periodic acid-Schiff sections using the color threshold function in ImageJ (National Institute of Health, Bethesda, MD) to create a binary image.

Determination of serum IgE. Mice were killed by avertin overdose and blood was collected via cardiac puncture. Serum was separated from whole blood by centrifugation after clotting overnight at $4{ }^{\circ} \mathrm{C}$. Enzymelinked immunosorbent assay for total serum IgE was performed according to the manufacturer's instructions (BD Biosciences).

Isolation of lymphocytes. LNs were collected from mice and passed through a $70 \mu \mathrm{M}$ strainer to obtain a suspension of free cells. Lamina propria cells were isolated by digestion in $0.5 \mathrm{mg} \mathrm{ml}^{-1}$ collagenase (Sigma-Aldrich) and $0.05 \%$ DNAse (StemCell Technologies, Vancouver, BC, Canada) in Dulbecco's modified Eagle's medium (DMEM) for $30 \mathrm{~min}$. Leukocytes were enriched by Percoll separation. Cells were cultured for up to 4 days in Th2 conditions: DMEM supplemented with $10 \%$ fetal bovine serum, $2 \mathrm{~mm}$ glutamine, $1 \%$ penicillin/ streptomycin, $25 \mathrm{~mm}$ HEPES, and $5 \times 10^{-5} \mathrm{M} 2$-mercaptoethanol with $1 \mu \mathrm{g} \mathrm{ml}^{-1}$ each anti-CD3 (145-2C11) and anti-CD28 (37.51) in the presence of anti-interferon- $\gamma\left(10 \mu \mathrm{g} \mathrm{ml}^{-1}\right)$, and IL4 $\left(80 \mathrm{ng} \mathrm{ml}^{-1}\right)$, and then stained with antibodies against CD45 and CD4 and analyzed by flow cytometry. Alternatively, cells isolated from LNs were not incubated and were prepared directly for flow cytometry.

Isolation of DCs. Flt3L-producing B16 melanoma cells were grown in vitro in complete DMEM supplemented with $10 \%$ fetal bovine serum and $1 \%$ penicillin/streptomycin. $5 \times 10^{4}$ cells were injected subcutaneously into mice. After tumor growth to $\sim 1 \mathrm{~cm}$ diameter, splenic DCs were isolated using a CD11c-positive selection kit (StemCell Technologies). The cell purity was verified by flow cytometry and found to be between 96 and $98 \%$.

Transcriptome analysis. DCs were incubated for $3 \mathrm{~h}$ with or without $50 \mu \mathrm{m}$ butyrate in complete DMEM, buffered at $\mathrm{pH} 7$ with bicarbonate, supplemented with $10 \%$ fetal bovine serum and $1 \%$ penicillin/ streptomycin. Cells were then stimulated with $100 \mathrm{ng} \mathrm{ml}^{-1}$ of LPS for $18 \mathrm{~h}$. Total RNA was extracted using an RNeasy Mini Kit according to the manufacturer's instructions (Qiagen). Approximately $500 \mathrm{ng}$ of RNA was prepped with the Truseq Stranded mRNA kit from Illumina (San Diego, CA) and sequenced on an Illumina MiSeq $75 \times 75$ Paired End v3 run. Using TopHat2, reads were aligned to the mm10 transcript reference and Cufflinks was used to determine differential expression. Differential expression tables were analyzed by the "core analysis" function in Ingenuity Pathways Analysis (Qiagen). Heat maps were generated using the open source $\mathrm{R}$ and VisR platforms. ${ }^{50}$
DC in vivo migration assay. DC migration to the lymph nodes upon antigen acquisition was tested in vivo via intranasal administration of $50 \mu \mathrm{l}$ DQ-OVA (Thermo Fisher Scientific, Waltham, MA). The percentage of FITC $^{+}$DCs in the mediastinal nodes was determined $24 \mathrm{~h}$ after the administration of FITC-OVA via flow cytometry using antibodies to CD11c.

T-cell activation assay. Flt3L-derived splenic DCs were incubated for $3 \mathrm{~h}$ with or without $50 \mu \mathrm{m}$ sodium butyrate in the culture media (complete DMEM supplemented with $10 \%$ fetal bovine serum, $2 \mathrm{mM}$ glutamine, $1 \%$ penicillin/ streptomycin, $25 \mathrm{~mm}$ HEPES, and $5 \times 10^{-5}$ M2-mercaptoethanol) and stimulated with $100 \mu \mathrm{g} \mathrm{ml}^{-1}$ OVA peptide (Worthington Biochemical (Lakewood, NJ)) in the presence of $100 \mathrm{ng} \mathrm{ml}^{-1}$ LPS. DCs were then washed extensively. This was followed by the addition of CFSE (Invitrogen (Carlsbad, CA))-labeled OTII CD4 ${ }^{+} \mathrm{T}$ cells $\left(10: 1 \mathrm{~T}\right.$-cell/DC ratio) for 7 days. CD4 ${ }^{+} \mathrm{T}$ cells were isolated from spleen by negative selection using RoboSep (StemCell Technologies). Purities were routinely 90-95\% CD4 ${ }^{+}$cells.

DC transwell migration assay. DCs were added to in the upper chamber of a $6 \mu \mathrm{m}$ pore transwell (Corning, Corning, NY). CCL19 was added in the lower chamber as a chemoattractant. Chambers were incubated at $37^{\circ} \mathrm{C}+5 \% \mathrm{CO}_{2}$ for $3 \mathrm{~h}$. Cells in lower chambers were recovered and counted by flow cytometry.

Statistics. Unless otherwise specified, differences between treatment groups were compared using paired and unpaired Student's $t$-test, as appropriate, or one-way analysis of variance (version 4.0, GraphPad Prism software, San Diego, CA).

SUPPLEMENTARY MATERIAL is linked to the online version of the paper at http://www.nature.com/mi

\section{ACKNOWLEDGMENTS}

We thank the BRC Animal Care facility; Taka Murakami of the BRC Genotyping facility; Les Rollins, Rupinder Dhesi, and Michael Williams of the BRC core staff; and Ingrid Barta of the BRC histology facility.

\section{AUTHOR CONTRIBUTIONS}

A.C. and M.R.H. conceived and initiated the project, planned and performed the experiments, analyzed the data, and wrote the manuscript. F.A. planned and performed experiments, and analyzed the data. P.A.D. and K.R.M. assisted with data analysis. L.A.R. planned and performed experiments. J.C., L.H., and J.M. performed experiments. C.Z., K.M.M., B.B.F., and W.W.M. co-supervised the study. C.Z., K.M.M., and W.W.M. edited the manuscript.

\section{DISCLOSURE}

The authors declared no conflict of interest.

(c) 2018 Society for Mucosal Immunology

\section{REFERENCES}

1. Miller, T. \& Wolin, M. Fermentations by saccharolytic intestinal bacteria. Am. J. Clin. Nutr. 32, 164-172 (1979)

2. Cummings, J. \& Macfarlane, G. The control and consequences of bacterial fermentation in the human colon. J. Appl. Bacteriol. 70, 443-459 (1991).

3. Wong, J. et al. Colonic health: fermentation and short chain fatty acids. J. Clin. Gastroenterol. 40, 235-243 (2006).

4. Høverstad, T. \& Midtvedt, T. Short-chain fatty acids in germfree mice and rats. J. Nutr. 116, 1772-1776 (1986).

5. Corrêa-Oliveira, R. et al. Regulation of immune cell function by short-chain fatty acids. Clin. Transl. Immunol. 5, e73 (2016).

6. Maslowski, K. et al. Regulation of inflammatory responses by gut microbiota and chemoattractant receptor GPR43. Nature 461, 12821286 (2009).

7. Trompette, A. et al. Gut microbiota metabolism of dietary fiber influences allergic airway disease and hematopoiesis. Nat. Med. 20, 159-166 (2014). 
8. Arrieta, M.-C. et al. Early infancy microbial and metabolic alterations affect risk of childhood asthma. Sci. Transl. Med. 7, 307ra152 (2015).

9. Russell, S.L. etal. Early life antibiotic-driven changes in microbiota enhance susceptibility to allergic asthma. EMBO Rep. 13, 440-447 (2012).

10. Ho, K.J. et al. Vancomycin treatment and butyrate supplementation modulate gut microbe composition and severity of neointimal hyperplasia after arterial injury. Physiol. Rep. 3, e12627 (2015).

11. Bender, A. et al. Colonic fermentation as affected by antibiotics and acidic pH: application of an in vitro model. Z. Gastroenterol. 39, 911-918 (2001).

12. Barcenilla, A. et al. Phylogenetic relationships of butyrate-producing bacteria from the human gut. Appl. Environ. Microbiol. 66, 1654-1661 (2000).

13. Pryde, S.E. et al. The microbiology of butyrate formation in the human colon. FEMS Microbiol. Lett. 217, 133-139 (2002).

14. Louis, P. \& Flint, H.J. Diversity, metabolism and microbial ecology of butyrate-producing bacteria from the human large intestine. FEMS Microbiol. Lett. 294, 1-8 (2009).

15. Vital, M., Howe, A.C. \& Tiedje, J.M. Revealing the bacterial butyrate synthesis pathways by analyzing (meta) genomic data. MBio 5, e00889 (2014).

16. Anandasabapathy, N. et al. Classical Flt3L-dependent dendritic cells control immunity to protein vaccine. J. Exp. Med. 211, 1875-1891 (2014).

17. Kaneko, T. et al. Growth stimulator for bifidobacteria produced by Propionibacterium freudenreichii and several intestinal bacteria. J. Dairy Sci. 77, 393-404 (1994).

18. Furusawa, $\mathrm{Y}$. et al. Commensal microbe-derived butyrate induces the differentiation of colonic regulatory T cells. Nature 504, 446-450 (2013).

19. Cahenzli, J. et al. Intestinal microbial diversity during early-life colonization shapes long-term IgE levels. Cell Host Microbe 14, 559-570 (2013).

20. Oeser, K. et al. Tcells are the critical source of IL-4/IL-13 in a mouse model of allergic asthma. Allergy 70, 1440-1449 (2015).

21. Mohrs, K. et al. A two-step process for cytokine production revealed by IL-4 dual-reporter mice. Immunity 23, 419-429 (2005).

22. Janeway, C.A., Travers, P., Walport, M. \& Shlomchik, M. Immunobiology, 5th edn. (Garland Science, New York, 2016).

23. Steinman, R.M. Dendritic cells and the control of immunity. Exp. Hematol. 26, 681-681 (1998).

24. Nastasi, C. et al. The effect of short-chain fatty acids on human monocytederived dendritic cells. Sci. Rep. 5, 16148 (2015).

25. Phythian-Adams, A.T. et al. CD11c depletion severely disrupts Th2 induction and development in vivo. J. Exp. Med. 207, 2089-2096 (2010).

26. Hammad, H. et al. Inflammatory dendritic cells-not basophils-are necessary and sufficient for induction of Th2 immunity to inhaled house dust mite allergen. J. Exp. Med. 207, 2097-2111 (2010).

27. Plantinga, M. et al. Conventional and monocyte-derived CD11b(+) dendritic cells initiate and maintain T helper 2 cell-mediated immunity to house dust mite allergen. Immunity 38, 322-335 (2013).

28. Blanchet, M.R. et al. CD34 is required for dendritic cell trafficking and pathology in murine hypersensitivity pneumonitis. Am. J. Respir. Crit. Care Med. 184, 687-698 (2011).

29. Mach, N. et al. Differences in dendritic cells stimulated in vivo by tumors engineered to secrete granulocyte-macrophage colony-stimulating factor or Flt3-ligand. Cancer Res. 60, 3239-3246 (2000).

30. Shurin, M.R. et al. FLT3 ligand induces the generation of functionally active dendritic cells in mice. Cell Immunol. 179, 174-184 (1997).
31. Arpaia, N. et al. Metabolites produced by commensal bacteria promote peripheral regulatory T-cell generation. Nature 504, 451-455 (2013).

32. Wang, B. et al. Butyrate inhibits functional differentiation of human monocyte-derived dendritic cells. Cell. Immunol. 253, 54-58 (2008).

33. Berndt, B. et al. Butyrate increases IL-23 production by stimulated dendritic cells. Am. J. Physiol. Gastrointest. Liver Physiol. 303, 92 (2012).

34. Frikeche, J. et al. Impact of valproic acid on dendritic cells function. Immunobiology 217, 704-710 (2012).

35. Randolph, G.J., Angeli, V. \& Swartz, M.A. Dendritic-cell trafficking to lymph nodes through lymphatic vessels. Nat. Rev. Immunol. 5, 617-628 (2005).

36. Lapin, B. et al. Relationship between prenatal antibiotic use and asthma in at-risk children. Ann. Allergy Asthma Immunol. 114, 203-207 (2015).

37. Sun, W.J. et al. Early-life antibiotic use is associated with wheezing among children with high atopic risk: a prospective European study. J. Asthma 52, 647-652 (2015).

38. Mulder, B. et al. Antibiotic use during pregnancy and asthma in preschool children: the influence of confounding. Clin. Exp. Allergy 46, 1214-1226 (2016).

39. Arpaia, N. et al. Metabolites produced by commensal bacteria promote peripheral regulatory T-cell generation. Nature 504, 451-455 (2013).

40. Hill, D.A. et al. Commensal bacteria-derived signals regulate basophil hematopoiesis and allergic inflammation. Nat. Med. 18, 538546 (2012).

41. Olszak, T. et al. Microbial exposure during early life has persistent effects on natural killer T cell function. Science 336, 489-493 (2012).

42. Lynch, S.V. et al. Effects of early-life exposure to allergens and bacteria on recurrent wheeze and atopy in urban children. J. Allergy Clin. Immunol. 134, 593- + (2014).

43. Zeissig, S. \& Blumberg, R.S. Life at the beginning: perturbation of the microbiota by antibiotics in early life and its role in health and disease. Nat. Immunol. 15, 307-310 (2014).

44. Durkin, H.G., Bazin, H. \& Waksman, B.H. Origin and fate of IgE-bearing lymphocytes. I. Peyer's patches as differentiation site of cells. Simultaneously bearing IgA and IgE. J. Exp. Med. 154, 640-648 (1981).

45. Auci, D.L. et al. Origin and fate of IgE-bearing lymphocytes. II. Gutassociated lymphoid tissue as sites of first appearance of lgE-bearing B lymphocytes and hapten-specific IgE antibody-forming cells in mice immunized with benzylpenicilloyl-keyhole limpet hemocyanin by various routes: relation to asialo GM1 ganglioside + cells and IgE/CD23 immune complexes. J. Immunol. 149, 2241-2248 (1992).

46. Schloss, P.D. et al. Introducing mothur: open-source, platform-independent, community-supported software for describing and comparing microbial communities. Appl. Environ. Microbiol. 75, 7537-7541 (2009).

47. Pruesse, E. et al. SILVA: a comprehensive online resource for quality checked and aligned ribosomal RNA sequence data compatible with ARB. Nucleic Acids Res. 35, 7188-7196 (2007).

48. McMurdie, P.J. \& Holmes, S. phyloseq: an R package for reproducible interactive analysis and graphics of microbiome census data. PLOS ONE 8, e61217 (2013).

49. DeBruin, E.J. et al. Mast cells in human health and disease. Methods Mol. Biol. 1220, 93-119 (2015).

50. Younesy, $H$. et al. VisRseq: R-based visual framework for analysis of sequencing data. BMC Bioinformatics 16 (Suppl 11), S2 (2015). 